\title{
Dichromatic dark matter
}

\author{
Yang Bai, ${ }^{a, b}$ Meng Su ${ }^{d, e, 1}$ and Yue Zhao ${ }^{b, c}$ \\ ${ }^{a}$ Department of Physics, University of Wisconsin, \\ 1150 University Avenue, Madison, WI 53706, U.S.A. \\ ${ }^{b}$ SLAC National Accelerator Laboratory, \\ 2575 Sand Hill Road, Menlo Park, CA 94025, U.S.A. \\ ${ }^{c}$ Stanford Institute for Theoretical Physics, Stanford University, \\ 382 Via Pueblo Mall, Varian Lab, Stanford, CA 94305, U.S.A. \\ ${ }^{d}$ Department of Physics, and Kavli Institute for Astrophysics and Space Research, \\ Massachusetts Institute of Technology, \\ 77 Massachusetts Avenue, Bldg. 37, Cambridge, MA 02139, U.S.A. \\ ${ }^{e}$ Institute for Theory and Computation, Harvard-Smithsonian Center for Astrophysics, \\ 60 Garden Street, MS-51, Cambridge, MA 02138, U.S.A. \\ E-mail: yangbai@physics.wisc.edu, mengsu@space.mit.edu, \\ zhaoyue@slac.stanford.edu
}

ABSTRACT: Both the robust INTEGRAL $511 \mathrm{keV}$ gamma-ray line and the recent tentative hint of the $135 \mathrm{GeV}$ gamma-ray line from Fermi-LAT have similar signal morphologies, and may be produced from the same dark matter annihilation. Motivated by this observation, we construct a dark matter model to explain both signals and to accommodate the two required annihilation cross sections that are different by more than six orders of magnitude. In our model, to generate the low-energy positrons for INTEGRAL, dark matter particles annihilate into a complex scalar that couples to photon via a charge-radius operator. The complex scalar contains an excited state decaying into the ground state plus an off-shell photon to generate a pair of positron and electron. Two charged particles with nondegenerate masses are necessary for generating this charge-radius operator. One charged particle is predicted to be long-lived and have a mass around $3.8 \mathrm{TeV}$ to explain the dark matter thermal relic abundance from its late decay. The other charged particle is predicted to have a mass below $1 \mathrm{TeV}$ given the ratio of the two signal cross sections. The $14 \mathrm{TeV}$ LHC will concretely test the main parameter space of this lighter charged particle.

KeYwords: Beyond Standard Model, Cosmology of Theories beyond the SM

ARXIV EPRINT: 1212.0864

\footnotetext{
${ }^{1}$ Einstein fellow.
} 


\section{Contents}

1 Introduction 1

2 Experimental data $\quad 3$

2.1 Experimental data 4

2.2 Dark matter annihilation cross sections for INTEGRAL and Fermi-LAT 4

3 Down-scattering excited dark matter $\quad 6$

$\begin{array}{lll}3.1 & \text { Operator analysis } & 7\end{array}$

$\begin{array}{ll}3.2 & \text { Renormalizable model } \\ 3.3 & \text { Dark }\end{array}$

$\begin{array}{ll}3.3 & \text { Dark matter relic abundance } \\ \end{array}$

4 Discussion and conclusions $\quad 15$

\section{Introduction}

Although dark matter serves as the dominant component of matter in our universe, its various properties remain unknown. From astrophysical evidence, there is no doubt that dark matter can interact with the Standard Model (SM) particles through gravitational interaction. However, whether there are additional interactions between dark matter and SM particles is still a mystery to us. Among several approaches to search for dark matter particles, measuring the cosmic ray spectrum provides the indirect detection of dark matter. Observing a high-energy gamma-ray line has long been believed to be the "smoking gun" of the dark matter detection [1-6]. Furthermore, the propagation of energetic photons in our Galaxy is less affected by the interstellar gas or Galactic magnetic field. The gamma-ray line signal can even provide the dark matter geometrical profile in our Galaxy.

The detection of celestial gamma-ray line at $511 \mathrm{keV}$ from the inner galaxy, which is believed to be caused by $e^{+} e^{-}$annihilations, was first reported by [7] and later confirmed by [8-11]. The total flux of the $511 \mathrm{keV}$ line has been estimated to be around $2 \times 10^{-3} \mathrm{~cm}^{-2} \mathrm{~s}^{-1}[12]$. About $97 \% e^{+} e^{-}$annihilations proceed through the intermediate state of a positronium atom, and $25 \%$ of these annihilations with opposite spin of $e^{+}$and $e^{-}$can produce $511 \mathrm{keV}$ line emission [13, 14]. Although this gamma-ray line has been known for decades, the identification of the positron source remains undetermined. Different astrophysical sources have been suggested during the years, but each of the models faces various challenge to explain the observations consistently. The relatively high ratio of the bulge to disk $511 \mathrm{keV}$ emission towards the inner Galaxy seems against its origin from hypernovae and gamma ray bursts, while the constraints on the production rate of high energy positrons also disfavors millisecond pulsars, as well as proton-proton collisions from e.g. microquasars, low luminosity X-ray binary jets, and the central supermassive black 
hole. Furthermore, pulsars, magnetars, and Galactic cosmic rays are not favored as major sources to the observed $511 \mathrm{keV}$ from the bulge, and stringent constraints on these origin of the $511 \mathrm{keV}$ line was suggested $[15,16]$.

Besides these astrophysical suggestions, the possibility that dark matter may create the $511 \mathrm{keV}$ gamma-ray line has been widely discussed, mainly motivated by the rather spheroidal, symmetric, bulge-centered morphology. The lack of higher energy gamma ray requires the injection energy of positrons to be below $\sim 3 \mathrm{MeV}$ [15]. This motivates studies for both $\mathrm{MeV}$-scale dark matter models [17-22] and $\mathrm{GeV} / \mathrm{TeV}$-scale dark matter models with a MeV mass splitting among different dark matter states [23-31]. ${ }^{1}$ Since TeV-scale dark matter with electroweak interaction strength can naturally gives correct thermal relic abundance, those models are more favored. Interestingly, the morphology of the $511 \mathrm{keV}$ signal profile has a peaked structure around the Galactic center, and the sharpness of the peak prefers to have dark matter annihilation rather than decaying as an explanation [12]. Thus we focus on the heavy dark matter scenario, and try to explain the $511 \mathrm{keV}$ INTEGRAL signal via dark matter annihilation.

One popular dark matter model to explain the INTEGRAL signal is the excited dark matter model with an $\mathrm{MeV}$ mass splitting [24]. This class of models suffer from the requirement of a large kinematic energy of dark matter to excite the ground state, hence relying on the Boltzmann tail of dark matter velocity distribution. It is under a debate whether the excited dark matter model can generate enough positrons to explain the large gamma-ray flux for the INTEGRAL data. In particular, [32] considers the scenario where both dark matter particles are excited, and [33] focuses on the one-side excited case. For the $100 \mathrm{GeV}$ dark matter mass region that we will consider in this paper, the situation is worse, because it requires a higher velocity to obtain enough kinematic energy comparing to a $\mathrm{TeV}$ mass dark matter. In our paper, we will address this problem and propose a new scenario of the Down-scattering excited Dark Matter (DeDM) to solve the Boltzmann suppression problem of the vanilla excited dark matter models.

More recently, the hint for another gamma-ray line around $130 \mathrm{GeV}$ from the Galactic center has been suggested by analyzing the public data from Fermi Gamma-ray Space Telescope (Fermi-LAT) [34, 35]. The hint becomes even stronger with the template fitting approach, which takes into account the spatial distribution of the LAT events towards the inner Galaxy along with the spectral information [36]. Fermi-LAT Collaboration has confirmed the hint of the peak at $\sim 130 \mathrm{GeV}$ using Pass 7 data. The peak shifts to a higher mass at $\sim 135 \mathrm{GeV}$ and the significance becomes weaker using the reprocessed Pass 7 data [37]. Such high energy gamma-ray line emission has been considered as a clean signature from dark matter annihilations. Many dark matter models have been constructed to explain the 130(135) GeV gamma-ray line feature (see [38] and references therein).

The morphology of the INTEGRAL $511 \mathrm{keV}$ and Fermi-LAT 130(135) GeV line shares similar structures: (1) the signal events concentrate at the center of the Galaxy with nondisk like distributions; (2) after smoothing Fermi-LAT signal with INTEGRAL's angular

\footnotetext{
${ }^{1}$ The down-scattering excited dark matter model, that this paper mainly focused on, was first discussed in $[29,30]$.
} 
resolution, they have comparable full widths at half maximum (FWHM) in both the longitudinal and latitude directions. This motivates us to explain both signals using same dark matter particle in our universe. The fittings for both signals prefer annihilation rather than decaying $[12,39]$. Having worked out the required annihilation cross sections, we find that the INTEGRAL $511 \mathrm{keV}$ cross section is six to seven orders of magnitude larger than that of the Fermi-LAT $130 \mathrm{GeV}$ line. This large hierarchy of cross sections sets a challenge when constructing a detailed model. However, the order of magnitude is comparable with an electromagnetic loop factor of $\mathcal{O}\left(\alpha^{2} / \pi^{2}\right)$ if the INTEGRAL and Fermi-LAT signals are coming from tree-level and loop-level processes, respectively. This serves as a clue for our model building.

Our paper is organized as following. In section 2, we emphasize the similarities of morphologies for both signals and work out the required cross sections. In section 3 , we propose our model, Down-scattering excited Dark Matter model, to explain both signals. In section section 3.1, we first provide a general operator analysis to illustrate the essence of our model and calculate the scales of cutoffs of the effective operators. Then we build up a concrete UV-completion for the operator analysis in section 3.2. In section 3.3, we discuss the thermal history of our model. One charged particle needs to be long-lived in our UV model, so that we have a semi-natural model to explain the final dark matter relic abundance.

\section{Experimental data}

In this section, we discuss the INTEGRAL and Fermi-LAT oberservations in more detail. Since photon is not much affected during its propagation in the Galaxy, photon coming from dark matter annihilation can be used to determine the dark matter distribution in our Galaxy. However, there are subtleties on how to map the INTEGRAL $511 \mathrm{keV}$ gamma-ray line signal profile to the dark matter distribution profile. This is because the low-energy positrons that are generated from dark matter particles can propagate through the interstellar medium and annihilate with electrons to photons away from the production site and bias the inferred dark matter distribution from the $511 \mathrm{keV}$ line morphology. In this paper, we assume that the positron propagation is negligible comparing to the spatial resolution of the INTEGRAL, thus the dark matter profile can be estimated by measuring $511 \mathrm{keV}$ emission morphology. On the other hand, the Fermi-LAT 130(135) GeV photons could directly be generated from dark matter particles, and its morphology can therefore tell us the dark matter profile.

We first compare the morphologies of the INTEGRAL $511 \mathrm{keV}$ and Fermi-LAT 130(135) GeV lines. After smoothing the Fermi-LAT 130(135) GeV line using the angular resolution of INTEGRAL, we find the spatial distributions are comparable to each other. Furthermore, assuming that both signals are generated by dark matter annihilation, we estimate the annihilation cross sections for the two processes. They will serve as inputs for our model building in the rest of the paper. 

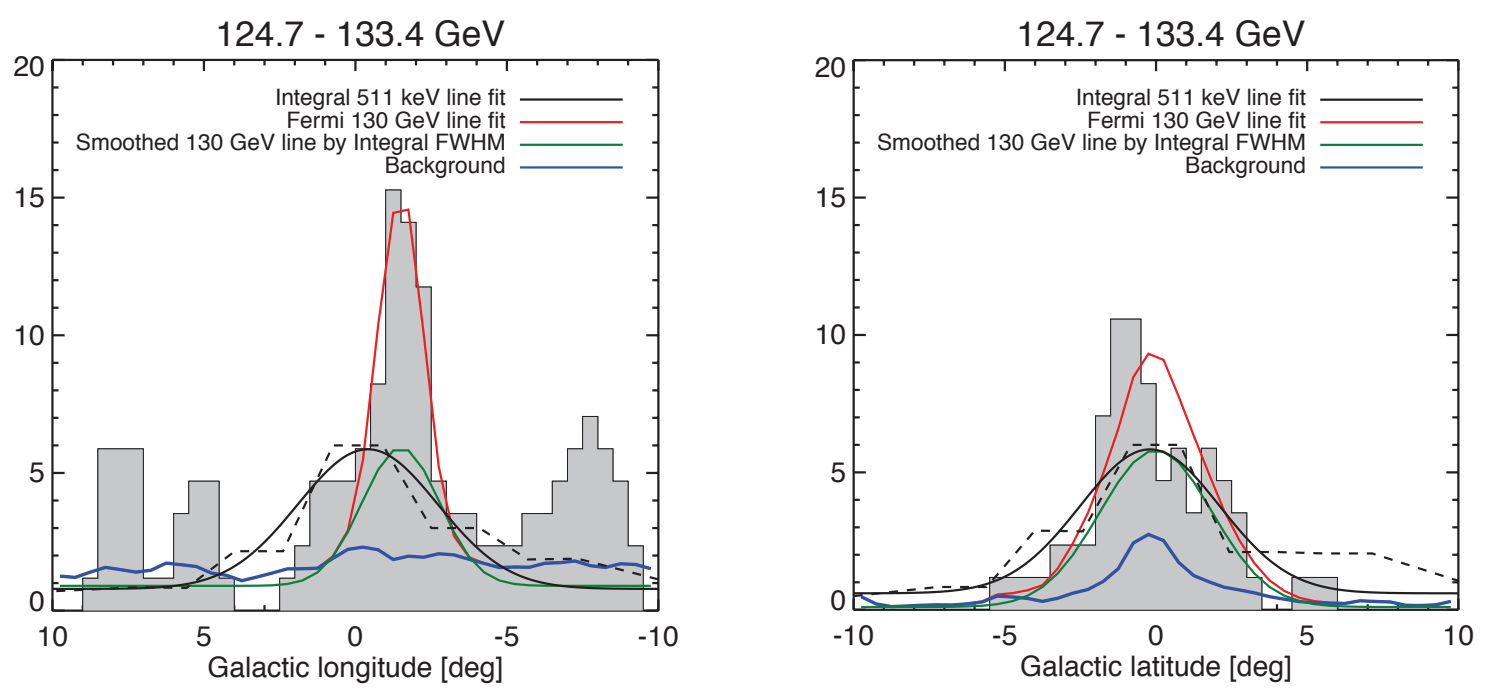

Figure 1. Comparison of the INTEGRAL $511 \mathrm{keV}$ line profile [40] and Fermi-LAT $130 \mathrm{GeV}$ line profile [41] from the Galacitc center, on longitudinal (left) and latitudinal (right) projections. The black dashed line shows the $511 \mathrm{keV}$ line profile measured by INTEGRAL, and the black solid line shows the fitted Gaussian. The shaded histogram shows the $130 \mathrm{GeV}$ line profile from 3.7 years of Fermi-LAT data. The red solid line shows the best fitted Gaussian of the $130 \mathrm{GeV}$ line, which is the same as figure 15 in [36]. The green solid line illustrates the $130 \mathrm{GeV}$ line profile smoothed by SPI $2.6^{\circ} \mathrm{FWHM}$ beam. For the INTEGRAL data, the vertical axis has an arbitrary scale in this plot.

\subsection{Experimental data}

Thanks to a coded mask located $1.7 \mathrm{~m}$ above the detector plane and a specific dithering observational strategy, the spectrometer (SPI) onboard the INTEGRAL observatory can image the sky with a spatial resolution of $\sim 2.6^{\circ}$ (FWHM). Based on observations recorded from February 22nd, 2003 to January, 2nd 2009, the study in [40] has obtained the morphology of the $511 \mathrm{keV}$ line towards the inner Galaxy. In figure 1, we compare the intensity of the $511 \mathrm{keV}$ gamma-ray line as a function of Galactic longitude and latitude with the $130 \mathrm{GeV}$ line profile obtained by fitting 3.7 years Fermi-LAT observations [41]. Especially, the dark green line shows the $130 \mathrm{GeV}$ line profile further smoothed by SPI $2.6^{\circ}$ FWHM beam.

Interestingly, both longitudinal and latitude distributions of INTEGRAL are comparable to those of Fermi-LAT after smoothing. Furthermore, both distributions show the tendency to be off-center in the negative longitudinal direction [36, 40]. These similarities motivate the attempts to build one dark matter model to explain both these two signals.

\subsection{Dark matter annihilation cross sections for INTEGRAL and Fermi-LAT}

As discussed in the previous section, both the $511 \mathrm{keV}$ line and the Fermi-LAT 130(135) $\mathrm{GeV}$ line could potentially be explained by dark matter annihilation. In this section, we estimate the required annihilation cross sections for both experiments. 
The gamma-ray line intensity in a given direction provided by dark matter annihilation is the line-of-sight integral of the squared dark matter number density along that given direction

$$
\frac{d \Phi_{\gamma}}{d E_{\gamma}}=d_{\chi} N_{\text {conv }} \frac{N_{e^{+} / \gamma}\left\langle\sigma v_{r}\right\rangle_{e^{+} / \gamma}}{2} \frac{R_{\odot} \rho_{\odot}^{2} J}{4 \pi m_{\chi}^{2}} \delta\left(E-E_{\gamma}\right)
$$

with the $J$-factor defined as:

$$
J=\int d b \int d \ell \int \frac{d s}{R_{\odot}} \cos b\left[\frac{\rho(r)}{\rho_{\odot}}\right]^{2}
$$

where $l$ and $b$ are longitude and latitude, and the integral of $s$ is along the line of sight. Here, $R_{\odot} \simeq 8.5 \mathrm{kpc}$ is the distance from the Sun to the galactic center; $\rho(r)$ is the dark matter halo profile; $\rho_{\odot} \simeq 0.4 \mathrm{GeV} \mathrm{cm}{ }^{-3}$ is the often-used dark matter density in the Solar system [42]; the relation between $r$ and $s$ is $r^{2}=s^{2}+R_{\odot}^{2}-2 s R_{\odot} \cos \ell \cos b ; N_{\gamma}$ $\left(N_{e^{+}}\right)$is the number of photons (positrons) generated from each dark matter annihilation hard process; $m_{\chi}$ is the dark matter mass; $\left\langle\sigma v_{r}\right\rangle_{e^{+}}$and $\left\langle\sigma v_{r}\right\rangle_{\gamma}$ are the annihilation cross sections. We define $d_{\chi}=1$ for self-conjugated dark matter, e.g. a real scalar or a Majorana fermion, and $d_{\chi}=\frac{1}{2}$ for a complex scalar or a Dirac fermion. $N_{\text {conv }}$ is the number of monochromatic photons that the final states could convert to. For Fermi-LAT, $N_{\text {conv }}=1$, since we assume that only monochromatic photons are produced in the hard process. For INTEGRAL, observations suggest that about $97 \%$ of positrons annihilate through positronium formation [43]. Only $1 / 4$ of annihilation takes place in the parapositronium state, which produces two $511 \mathrm{keV}$ photons. So, we have $N_{\text {conv }} \approx 0.55$ for INTEGRAL.

We consider both the Einasto and the Navarro-Frenk-White (NFW) dark matter profile

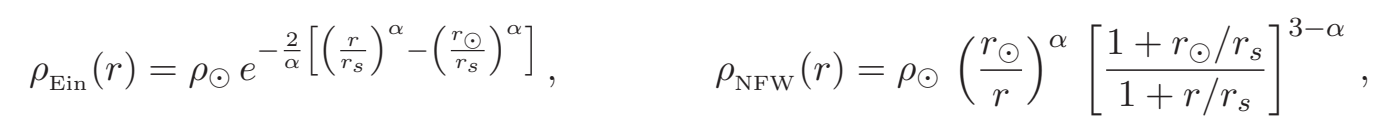

with $r_{s}=20 \mathrm{kpc}$ and $\alpha=0.17$ for Einasto [44] and $\alpha=1$ for NFW [45]. Using the fitted fluxes for the INTEGRAL signal (the dark matter+disk ones) in ref. [46], we obtain the annihilation cross sections as ${ }^{2}$

$$
\left\langle\sigma v_{r}\right\rangle_{\gamma, 511, \operatorname{Ein}(\mathrm{NFW})}=\frac{1}{d_{\chi} N_{e^{+}}} \times 1.5(0.28) \times 10^{5} \times\left(\frac{m_{\chi}}{100 \mathrm{GeV}}\right)^{2} \mathrm{pb} \cdot c .
$$

For the Fermi-LAT 130(135) GeV gamma line, we use the fitted fluxes from ref. [34] for both profiles to calculate the cross sections ${ }^{3}$

$$
\left\langle\sigma v_{r}\right\rangle_{\gamma, 135, \operatorname{Ein}(\mathrm{NFW})}=\frac{1}{d_{\chi} N_{\gamma}} \times 0.42(0.76) \times 10^{-1} \times\left(\frac{m_{\chi}}{100 \mathrm{GeV}}\right)^{2} \mathrm{pb} \cdot c .
$$

To quantify the ratio of the required cross sections for two experimental results, we define $R_{511}^{135} \equiv\left\langle\sigma v_{r}\right\rangle_{\gamma, 135} /\left\langle\sigma v_{r}\right\rangle_{\gamma, 511}$. Taking $N_{e^{+}}=N_{\gamma}=2$, we have the experimentally measured ratios as

$$
\left(R_{511}^{135}\right)_{\mathrm{exp}, \mathrm{Ein}} \approx 2.9 \times 10^{-7}, \quad\left(R_{511}^{135}\right)_{\mathrm{exp}, \mathrm{NFW}} \approx 2.7 \times 10^{-6},
$$

\footnotetext{
${ }^{2}$ Here, we use different parameters for dark matter profiles compared to the ones in ref. [46]. We simply rescale their signal flux by the ratio of $J$ functions, which could bring an uncertainty of $\mathcal{O}(1)$.

${ }^{3}$ The choice of the dark matter profile will affect the astrophysics background subtraction, thus $\frac{\sigma_{511, \text { Ein }}}{\sigma_{135, \operatorname{Ein}}} \neq$ $\frac{\sigma_{511, \mathrm{NFW}}}{\sigma_{135, \mathrm{NFW}}}$.
} 
where clearly show a large hierarchy for the two required cross sections. We want to also stress that the astrophysical uncertainties are fairly large and a global fit by combining the INTEGRAL and Fermi-LAT might bring the uncertainty down. The cross section ratio between these two expertiments is $\mathcal{O}\left(10^{-6} \sim 10^{-7}\right)$. This will be the input for model building in latter sections. Interestingly, this ratio is comparable to the square of the electromagnetic loop factor $\left(\alpha^{2} / \pi^{2}\right) \sim 6 \times 10^{-6}$, which implies these two experimental results may be related by a loop with two electromagnetic vertices. It serves as a clue for model building.

\section{Down-scattering excited dark matter}

There are several interesting features required to construct dark matter models if both signatures are to be explained by the same dark matter particle with a mass at the $100 \mathrm{GeV}$ scale.

- The required cross section for the INTEGRAL data is amazingly large. For a simplest estimation on the annihilation rate, we get $\sigma v \sim 1 /\left(4 \pi m_{\chi}^{2}\right) \sim 3 \times 10^{3} \mathrm{pb} \cdot c$ for $m_{\chi}$ around $100 \mathrm{GeV}$. This estimation is three orders of magnitude smaller than the required one. Additional mechanisms are therefore required to increase the annihilation rate. There are several ways to achieve this and we pay special attention on the resonance enhancement [47-53].

- To explain the INTEGRAL data, primary positron injections from dark matter are required. Since we don't see any excess for other cosmic rays, the underlying dark matter model should be arranged to treat positron/electron differently from other particles. In principle, this can be achieved either from kinematic constraints or symmetry reasons.

- The ratio of the two cross sections is $\left\langle\sigma v_{r}\right\rangle_{135} /\left\langle\sigma v_{r}\right\rangle_{511} \sim 10^{-7}$ or $10^{-6}$. The dark matter model should also provide a natural explanation for this hierarchy of two cross sections.

- The model should provide correct amount of dark matter relic abundance to be consistent with observation.

In the following, we will provide a particle physics model to incorporate all above four ingredients. Specifically, we will use a resonance particle in the $s$-channel to increase the dark matter annihilation cross section required for $511 \mathrm{keV}$ gamma-ray line. The kinematic constraints from a small mass splitting will be used in this paper to select positron/electron as the signals from dark matter annihilation. Instead of introducing a light mediator, e.g. dark photon, for the dark matter sector connecting to the positron/electron, we use photon as a more natural mediator to achieve this goal. Noticing that a neutral scalar field cannot decay into another neutral scalar field plus one on-shell photon, which is the reason why $\Upsilon[(n+1) S] \nrightarrow \Upsilon[n S]+\gamma$, a neutral scalar coupling to photon with the charge-radius operator can naturally induce $e^{ \pm}$without generating a photon signal in the meanwhile. The mass difference of the two scalar fields is chosen to be small such that the kinematic 
energy of $e^{ \pm}$is small enough to be consistent with observation. To explain the ratio of the cross sections, we will have the cross section for INTEGRAL to be controlled by coefficients of renormalizable operators, while loop-generated higher-dimensional operators for FermiLAT. We will first perform an operator analysis and then provide a UV-complete model.

\subsection{Operator analysis}

We introduce one Dirac fermion $\chi$ and one complex scalar field $\Phi \equiv\left(\phi_{1}+i \phi_{2}\right) / \sqrt{2}$ in the dark matter sector. Both $\chi$ and $\phi_{1}$ are stable particles and coexist in our current Universe. In our study, we will assume that the dark matter component $\chi$ occupies the majority of the dark matter energy, but we will come back to discuss the relative relic abundances of them later. The interactions of the dark matter sector to the SM particles are described by the following set of effective operators

$$
-\mathcal{L} \supset i \lambda_{\chi} \bar{\chi} \gamma^{5} \chi S+\mu S \Phi^{\dagger} \Phi+\frac{\lambda_{S} \alpha}{4 \pi M} S F_{\mu \nu} F^{\mu \nu}+\frac{\lambda_{\Phi} e}{16 \pi^{2} M^{2}} \partial_{\mu} \Phi \partial_{\nu} \Phi^{\dagger} F^{\mu \nu},
$$

where we implicitly assume that the higher-dimensional operators can be generated at oneloop level. The annihilation of $\chi$ 's is through exchanging the real scalar $S$ in the $s$-channel. For the INTEGRAL data, a small mass scale at around $1 \mathrm{MeV}$ is required to generate positrons almost at rest. In our model, we introduce this small mass scale as the mass splitting of $\phi_{1}$ and $\phi_{2}$ from $\Phi=\left(\phi_{1}+i \phi_{2}\right) / \sqrt{2}$ such that $\delta \equiv m_{\phi_{2}}-m_{\phi_{1}} \ll m_{\phi_{1}}$ and $\delta \sim$ $1 \mathrm{MeV}$. Noticing that the parameter $\delta$ explicitly breaks the global $\mathrm{U}(1)_{\phi}$, so the smallness of $\delta$ is technically natural. Expanding the last operator in terms of $\phi_{1}$ and $\phi_{2}$, we have

$$
\frac{\lambda_{\Phi} e}{16 \pi^{2} M^{2}} i \partial_{\mu} \phi_{2} \partial_{\nu} \phi_{1} F^{\mu \nu}
$$

Using the equation of motion, one can rewrite the above operator as $\phi_{2} \partial_{\nu} \phi_{1} \partial_{\mu} F^{\mu \nu}=$ $\phi_{2} \partial_{\nu} \phi_{1} j^{\nu}$. This indicates that $\phi_{2}$ cannot decay to a mass-on-shell photon. For $2 m_{e}<\delta<2 m_{\mu}$, we have the leading decay channel of $\phi_{2}$ as

$$
\phi_{2} \rightarrow \phi_{1}+\gamma^{*} \rightarrow \phi_{1}+e^{+}+e^{-} .
$$

Photon, naturally, behaves as a mediator for the dark matter sector to generate positrons.

The processes to generate positrons for INTEGRAL and photons for Fermi-LAT are shown in figure 2, where the solid thick points indicate higher-dimensional operators for those vertices. Although it looks like that the relative cross sections for those two processes are unrelated to each other, we will show in a concrete renormalizable model that the overall cross sections could have a relation in section 3.2. In order to generate slowly moving positron from dark matter annihilation, as preferred from the INTEGRAL data, there are two conditions required: (1) the mass splitting $\delta$ should be close to $2 m_{e}$; (2) $\phi_{2}$ cannot have a large boost. The first condition can be satisfied by choosing $\delta \gtrsim 2 m_{e}$. The second condition can be arranged by choosing $m_{\phi_{2}} \lesssim m_{\chi}$.

We first calculate the annihilation cross section for INTEGRAL. Using the interactions of $\phi_{2}$ in eq. (3.1), one gets the annihilation cross section of $\chi \bar{\chi} \rightarrow \phi_{2} \phi_{2}$ at leading order in $v_{r}$ as

$$
\left(\sigma v_{r}\right)_{\phi_{2} \phi_{2}}=\frac{\lambda_{\chi}^{2} \mu^{2}}{32 \pi} \frac{1}{\left(4 m_{\chi}^{2}+m_{\chi}^{2} v_{r}^{2}-m_{S}^{2}\right)^{2}+m_{S}^{2} \Gamma_{S}^{2}} \sqrt{1-\frac{m_{\phi_{2}}^{2}}{m_{\chi}^{2}}},
$$



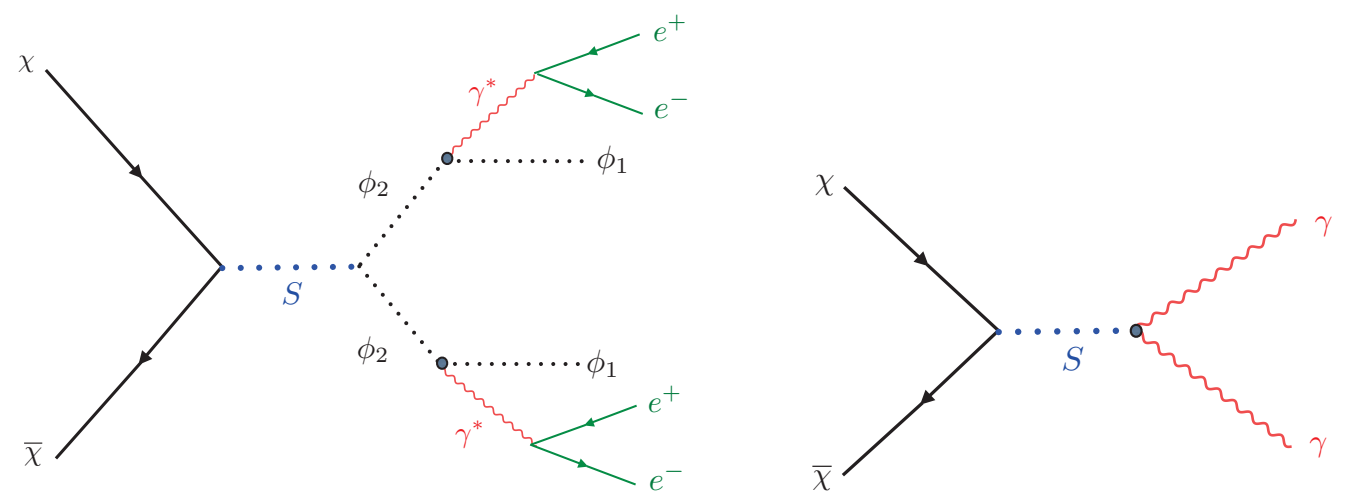

Figure 2. The Feynman diagrams for INTEGRAL (left) and Fermi-LAT (right).
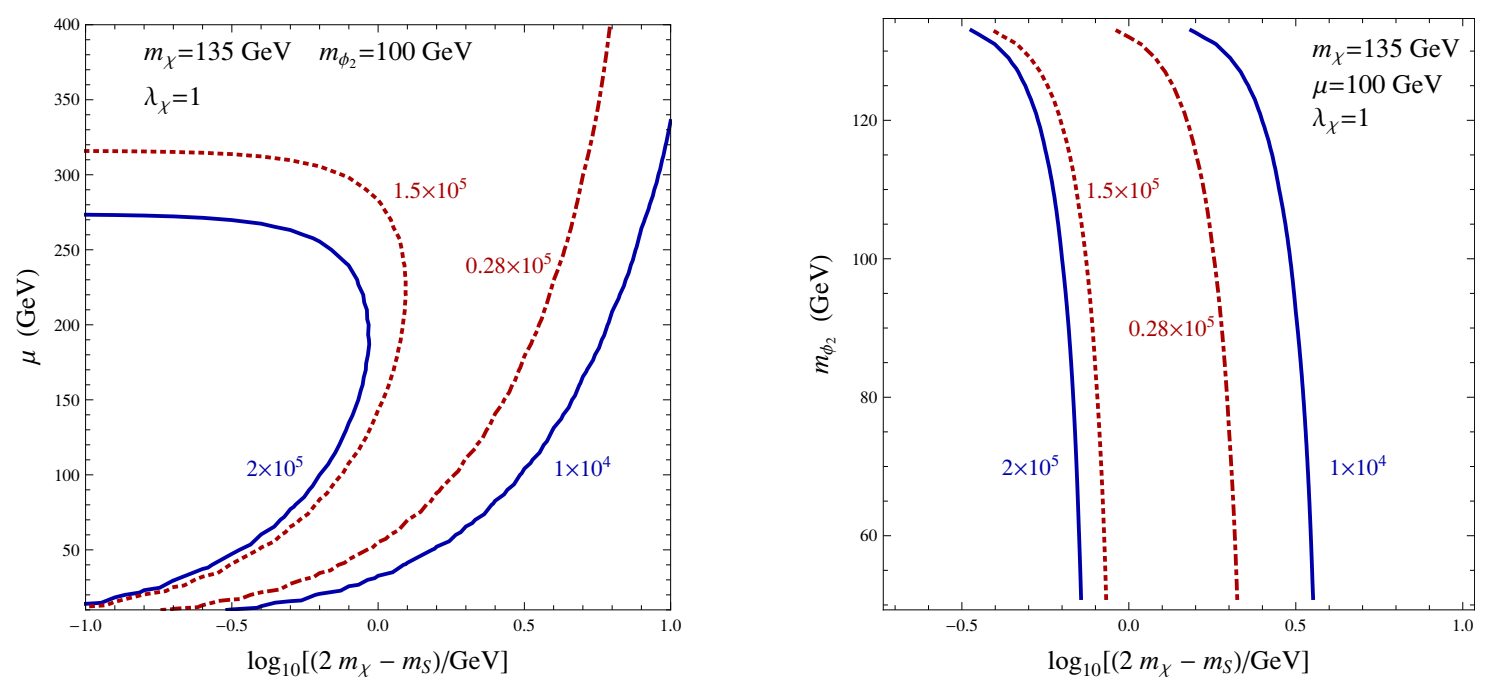

Figure 3. Left panel: the contour plots of the annihilation rates in $\mathrm{pb} \cdot \mathrm{c}$ for $\mu$ and the mass difference $\left(2 m_{\chi}-m_{S}\right)$. The red and dotted line is the required cross section to explain the INTEGRAL data for the Einasto profile, while the red and dotdashed line is for the NFW profile in eq. (2.4). Right panel: the same as the left one but in terms of $m_{\phi_{2}}$ and $\left(2 m_{\chi}-m_{S}\right)$.

We are interested in the parameter space with $2 m_{\chi}>m_{S}>2 m_{\phi_{2}}$. The decay width of $S$ is calculated to be $\Gamma_{S} \approx 2 \Gamma_{S}^{\phi_{2}}+\Gamma_{S}^{\gamma}$ with

$$
\begin{aligned}
\Gamma_{S}^{\phi_{2}} & =\frac{\mu^{2}}{32 \pi m_{S}} \sqrt{1-\frac{4 m_{\phi_{2}}^{2}}{m_{S}^{2}}} \\
\Gamma_{S}^{\gamma} & =\frac{\lambda_{S}^{2} \alpha^{2} m_{S}^{3}}{64 \pi^{3} M^{2}}
\end{aligned}
$$

Here we treat the decay width of $S \rightarrow \phi_{1} \phi_{1}$ to be approximately the same as $\Gamma_{S}^{\phi_{2}}$. 


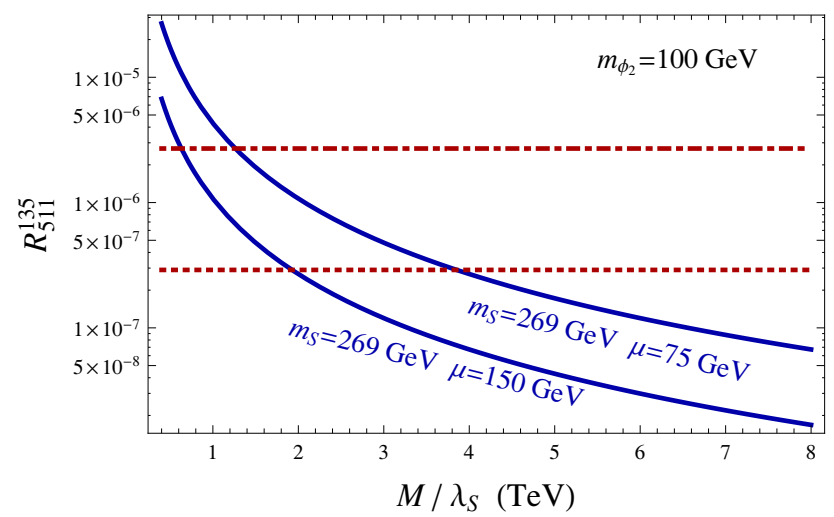

Figure 4. The ratio of annihilation rates for the Fermi-LAT and INTEGRAL signals as a function of the cutoff of the higher-dimensional operators. Here, we choose $m_{\phi_{2}}=100 \mathrm{GeV}$. The dotted and dot-dashed lines (red) indicate the approximate value from experimental measurements for two different dark matter profiles.

For INTEGRAL, we need to calculate the velocity-averaged annihilation rate, which is given by

$$
\left\langle\sigma v_{r}\right\rangle_{511}=\left\langle\left(\sigma v_{r}\right)_{\phi_{2} \phi_{2}}\left(v_{r}\right)\right\rangle=\frac{x^{3 / 2}}{\sqrt{4 \pi}} \int v_{r}^{2} d v_{r} e^{-x v_{r}^{2} / 4}\left(\sigma v_{r}\right)_{\phi_{2} \phi_{2}}\left(v_{r}\right)
$$

where $x=m_{\chi} / T=v_{0}^{-2}$ with $v_{0}$ determining the variance of the Gaussian dark matter velocity distribution. In our numerical calculation, we neglect the upper limit of the integration, which is controlled by the escaping velocity of dark matter in the galaxy and has only a small effect on our final results.

In figure 3, we show the contours of the annihilation rates of $\left\langle\sigma v_{r}\right\rangle_{511}$ in terms of $\mu$ and $\left(2 m_{\chi}-m_{S}\right)$ in the left panel, also $m_{\phi_{2}}$ and $\left(2 m_{\chi}-m_{S}\right)$ in the right panel. To obtain a large annihilation rate around $10^{5} \mathrm{pb}$ to explain the INTEGRAL data, the resonance mass has to be very close to twice of the dark matter mass. The mass splitting should be a few $\mathrm{GeV}$ for the parameter $\mu \sim 100 \mathrm{GeV}$. In figure 3, we only presented the results for $m_{S} \lesssim 2 m_{\chi}$. The case with $m_{S} \gtrsim 2 m_{\chi}$ has an additional contribution to the total width of $S$ from $S \rightarrow \chi \bar{\chi}$, and has similar results. From the right panel of figure 3, we can see that the annihilation rate is insensitive to $m_{\phi_{2}}$ except for the region with $m_{\phi_{2}} \sim m_{\chi}$. One might think that $\phi_{2}$ can be as light as possible. However, a light $\phi_{2}$ generated from dark matter annihilation can have a large Lorentz boost. As a consequence, $e^{+}$from $\phi_{2}$ decays is also boosted and too energetic to explain the INTEGRAL data [12]. Therefore, we restrict the parameter space in our later study to have $m_{\phi_{2}}$ at least above $50 \mathrm{GeV}$.

For the Fermi-LAT signal, instead of obtaining the absolute annihilation rate, we calculate the ratio of the required signal strengths for Fermi-LAT and INTEGRAL. The ratio is equal to the branching ratio of the two decay channels of $S$ in eq. (3.6), assuming that the additional contribution from the process $\phi_{1} \phi_{1} \rightarrow \gamma \gamma$ is small. By taking the ratio, 


\begin{tabular}{|c|cccc|}
\hline & spin & $\mathrm{U}(1)_{Y}$ & $\mathrm{U}(1)_{\phi}$ & $\mathbb{Z}_{2}$ \\
\hline$\chi$ & $\frac{1}{2}$ & 0 & 0 & - \\
$S$ & 0 & 0 & 0 & + \\
$\Phi=\frac{1}{\sqrt{2}}\left(\phi_{1}+i \phi_{2}\right)$ & 0 & 0 & 1 & - \\
$X_{1}$ & 0 & 1 & 1 & - \\
$X_{2}$ & 0 & 1 & 0 & + \\
\hline
\end{tabular}

Table 1. Matter content and corresponding charge assignments. The global symmetry $\mathrm{U}(1)_{\phi}$ is only an approximate one. The small mass splitting of $\phi_{1}$ and $\phi_{2}$ breaks it.

the dependence on the resonance propagator is cancelled and we have

$$
R_{511}^{135}=\frac{\lambda_{S}^{2} \alpha^{2} m_{S}^{4}}{2 \pi^{2} M^{2} \mu^{2}}\left(1-\frac{4 m_{\phi_{2}}^{2}}{m_{S}^{2}}\right)^{-1 / 2}
$$

We show this ratio of the annihilation rates in figure 4 by fixing $m_{\phi_{2}}=100 \mathrm{GeV}$. From figure 4, we can find that the cutoff of the operators in eq. (3.1) should be several TeV.

From the effective operator analysis in this section, we have seen that it is possible to explain the required annihilation rates for both INTEGRAL and Fermi-LAT. Our model is economical in a sense that only a few operators and a small number of degrees of freedom are required to explain the data. On the other hand, we should also admit that the resonance requirement of $\left(2 m_{\chi}-m_{S}\right) \ll m_{S}$ is a tuning point of the parameter space of the current model. Additional ingredients are therefore required to explain this delicate mass relation. We leave this direction of exploration to a future study. Here we emphasize that the ratio of INTEGRAL and Fermi-LAT signals are independent on the way we enhance the annihilation cross sections. Thus one can attach the rest of the model to any other ways of enhancement, e.g. a light mediator in the $t$-channel plus the Sommerfeld enhancement [54]. In the next section, we construct a renormalizable model to UV complete the Lagrangian in eq. (3.1) and explain the common origin of the last two operators in eq. (3.1).

\subsection{Renormalizable model}

One way to UV complete the effective Lagrangian in the previous section is to introduce electromagnetic charged states to connect the dark matter sector to photon. In order to have the state $\phi_{1}$ stable, at least two charged particles are required to preserve the discrete symmetry associated with $\phi_{1}$. As one example, we introduce two charged complex scalar fields, $X_{1}$ and $X_{2}$. One could also study fermionic charged states in the same procedure. Under $\mathrm{U}(1)_{Y}$ or $\mathrm{U}(1)_{e m}$ after electroweak symmetry breaking, $X_{1}$ and $X_{2}$ have charge one. The global symmetries that we introduce contain a $\mathcal{Z}_{2}$ symmetry responsible for the stability of the dark matter particles and a $\mathrm{U}(1)_{\phi}$ protecting the mass degeneracy of $\phi_{1}$ and $\phi_{2}$. We show the field content and symmetries in table 1. 
Based on the symmetries in table 1, we have the following subset of operators allowed by the symmetries,

$$
\begin{aligned}
-\mathcal{L} \supset i \lambda_{\chi} \bar{\chi} \gamma^{5} \chi S+\mu S \Phi^{\dagger} \Phi+\mu_{1}\left(\Phi X_{1} X_{2}^{\dagger}+\Phi^{\dagger} X_{1}^{\dagger} X_{2}\right)+\mu_{2} S X_{1} X_{1}^{\dagger}+\mu_{3} S X_{2} X_{2}^{\dagger}+\lambda_{1} \Phi \Phi^{\dagger} H H^{\dagger} \\
\quad+\frac{1}{2} m_{S}^{2} S^{2}+m_{\phi}^{2} \Phi \Phi^{\dagger}+\frac{1}{2} m_{\phi} \delta\left(\Phi^{2}+\Phi^{\dagger 2}\right)+m_{X_{1}}^{2} X_{1} X_{1}^{\dagger}+m_{X_{2}}^{2} X_{2} X_{2}^{\dagger} \\
+\lambda_{e}^{i} X_{1} \bar{\chi} e_{R}^{i}+\text { h.c. }
\end{aligned}
$$

Here we only list the operators which are relevant to the processes we concern in this paper. Especially the operator $S H H^{\dagger}$ is neglected, which is assumed to have a small coefficient. The last operator generically introduces lepton flavor violation processes, so the couplings $\lambda_{e}^{i}$ ( $i$ is the flavor index) should be small.

We first note that the vertices $\left(\Phi X_{1} X_{2}^{\dagger}+\Phi^{\dagger} X_{1}^{\dagger} X_{2}\right)$ could generate the charge radius operator for the $\Phi$ field as shown in the last operator in eq. (3.1). After a calculation of the triangle diagram with $X_{1}$ and $X_{2}$ propagating in the loop, we get the Feynman rule of the following operator $\partial_{\mu} \Phi \partial_{\nu} \Phi^{\dagger} F^{\mu \nu}=i \partial_{\mu} \phi_{2} \partial_{\nu} \phi_{1} F^{\mu \nu}$

$$
\frac{e \mu_{1}^{2}}{32 \pi^{2}} \int_{0}^{1} d x \int_{0}^{1-x} d y\left[\frac{(1-2 x) k_{1}^{\alpha}+(1-2 y) k_{2}^{\alpha}}{(1-x-y) m_{X_{2}}^{2}+(x+y) m_{X_{1}}^{2}+\left(x k_{1}+y k_{2}\right)^{2}-x k_{1}^{2}-y k_{2}^{2}}-\left(m_{X_{1}} \leftrightarrow m_{X_{2}}\right)\right] \text {, }
$$

where $k_{1}$ and $k_{2}$ are momenta of $\phi_{1}$ and $\phi_{2}$ with opposite directions towards the vertex, and $\alpha$ is the Lorentz index of the photon. In the limit $m_{X_{1,2}}^{2} \gg k_{1}^{2}, k_{2}^{2}$, we can match to the coefficient of the effective operator in eq. (3.1) as

$$
\frac{\lambda_{\Phi}}{M^{2}}=\frac{\mu_{1}^{2}\left[3\left(m_{X_{1}}^{4}-m_{X_{2}}^{4}\right)-2\left(m_{X_{1}}^{4}+4 m_{X_{1}}^{2} m_{X_{2}}^{2}+m_{X_{2}}^{4}\right) \log \left(m_{X_{1}} / m_{X_{2}}\right)\right]}{6\left(m_{X_{1}}^{2}-m_{X_{2}}^{2}\right)^{4}} .
$$

We notice that the above formula vanishes when $m_{X_{1}}=m_{X_{2}}$. This can be understood by the enhanced discrete symmetry, $\Phi \rightarrow \Phi^{\dagger}, X_{1} \leftrightarrow X_{2}$, in the Lagrangian when $X_{1}$ and $X_{2}$ have degenerate masses. ${ }^{4}$ The charge-radius operator violates this discrete symmetry, thus cannot be generated when $m_{X_{1}}=m_{X_{2}}$. Another more intuitive explanation is to think $\Phi$ as a composite particle of $X_{1}^{+}$and $X_{2}^{-}$. If the mass of $X_{2}^{-}$is much heavier than $X_{1}^{+}$, one can treat $X_{1}^{+}$as a particle rotating around $X_{2}^{-}$and have a nonzero charge radius. However, for the mass degenerate case, $X_{1}^{+}$and $X_{2}^{-}$should be treated with equal foot and rotate around the center with the same radius. As a result, for each orbit the net charge is zero and the charge radius is zero.

Similarly, we can integrate out $X_{1}$ and $X_{2}$ to generate the effective operator coupling $S$ to two photons. To match the coefficient in eq. (3.1), we have

$$
\frac{\lambda_{S}}{M}=\frac{1}{12}\left(\frac{\mu_{2}}{m_{X_{1}}^{2}}+\frac{\mu_{3}}{m_{X_{2}}^{2}}\right) .
$$

In the limit $m_{X_{2}}^{2} / \mu_{3} \ll m_{X_{1}}^{2} / \mu_{2}$, we have

$$
m_{X_{2}} \approx 410 \mathrm{GeV} \times\left(\frac{M / \lambda_{S}}{2 \mathrm{TeV}}\right)^{1 / 2}\left(\frac{\mu_{3}}{1 \mathrm{TeV}}\right)^{1 / 2} .
$$

\footnotetext{
${ }^{4}$ Operator $\lambda_{e}^{i} X_{1} \bar{\chi} e_{R}^{i}$ does not preserve this symmetry, but this operator could have a very small coefficient and is irrelevant to this calculation.
} 
Using the values of $M / \lambda_{S}$ in figure 4 , we anticipate at least the charged particle $X_{2}$ to have a mass below $1 \mathrm{TeV}$. This charged particle $X_{2}$ can decay into one lepton plus one neutrino, for example via the higher dimensional operator $X_{2} \tilde{H} \bar{L} e_{R}$.

\subsection{Dark matter relic abundance}

In our DeDM model, we have two stable particles in our spectrum: $\chi$ and $\phi_{1}$. In our previous analysis, we have assumed that the majority of dark matter in our universe is mainly composed of $\chi$. To justify our assumption, it is important to study the thermal history of $\chi$ and $\phi_{1}$. In this section, we demonstrate that our setup contains enough ingredients to induce a right relic abundance for $\chi$, thus it could be the dominant part of the dark matter in our current Universe.

The thermal relic abundance of $\phi_{1}$ is controlled by the parameter $\lambda_{1}$ in eq. (3.9), which is similar to the "Higgs portal" dark matter models $[55,56]$. For $m_{\phi_{1}}<m_{h}$, the main annihilation cross section is [57]

$$
\sigma v_{r}\left(\phi_{1}\right)=\frac{2 \lambda_{1}^{2} v_{\mathrm{EW}}^{2}}{\left(4 m_{\phi_{1}}^{2}-m_{h}^{2}\right)^{2}+m_{h}^{2} \Gamma_{h}^{2}\left(m_{h}\right)} \frac{\Gamma_{h}\left(2 m_{\phi_{1}}\right)}{2 m_{\phi_{1}}},
$$

where $v_{\mathrm{EW}}=246 \mathrm{GeV}$ is the electroweak vacuum expectation value. The function $\Gamma_{h}(m)$ is the width of a Higgs boson in the SM with a mass at $m$. For $\lambda_{1}=1, m_{\phi_{1}}=100 \mathrm{GeV}$ and $m_{h}=125 \mathrm{GeV}$, we have $\sigma v_{r}\left(\phi_{1}\right) \approx 581 \mathrm{pb}$ and $\Omega_{\phi_{1}} h^{2} \approx 1.4 \times 10^{-3} \times \Omega_{\mathrm{DM}} h^{2}$. Thus the relic abundance of $\phi_{1}$ can be naturally small.

To satisfy the dark matter relic abundance, a non-trivial thermal history of $\chi$ is needed. This is because a large annihilation cross section in eq. (3.4) is needed to explain the INTEGRAL data. The thermal relic abundance of $\chi$ is very small compared to the required dark matter energy density. Noticing that the last operator in eq. (3.9) can introduce the decay channel, $X_{1}^{+} \rightarrow \chi e^{+}$, the late decay of thermally abundant $X_{1}^{+}$particles can generate enough $\chi$, and therefore explain why $\chi$ could be the majority of dark matter.

We first calculate the thermal relic abundance of the charged particle $X_{1}^{ \pm}$before it decays into $\chi$ and a positron/electron. There are two classes of annihilation channels for $X_{1}^{ \pm}$. The first class has a photon or $Z$ boson exchanging in the $s$-channel with final states as a pair of the SM fermions, $W^{+} W^{-}$gauge bosons, and $h Z$. The second class includes the $t$-channel diagrams, interfering with seagull diagrams. Assuming that the mass $X_{1}^{ \pm}$is far above the SM particle masses and neglecting the SM particle masses, we have

$$
\begin{gathered}
\sigma v_{r}\left(X_{1}^{+} X_{1}^{-} \rightarrow \gamma \gamma\right)=\frac{e^{4}}{8 \pi m_{X_{1}}^{2}}, \quad \sigma v_{r}\left(X_{1}^{+} X_{1}^{-} \rightarrow Z Z\right)=\frac{e^{4} s_{W}^{4}}{8 \pi c_{W}^{4} m_{X_{1}}^{2}}, \\
\sigma v_{r}\left(X_{1}^{+} X_{1}^{-} \rightarrow \gamma Z\right)=\frac{3 e^{4} s_{W}^{2}}{4 \pi c_{W}^{2} m_{X_{1}}^{2}}, \quad \sigma v_{r}\left(X_{1}^{+} X_{1}^{-} \rightarrow h Z\right)=\frac{e^{4}}{1536 \pi c_{W}^{4} m_{X_{1}}^{2}} v_{r}^{2}, \\
\sigma v_{r}\left(X_{1}^{+} X_{1}^{-} \rightarrow W^{+} W^{-}\right)=\frac{e^{4}}{1536 \pi c_{W}^{4} m_{X_{1}}^{2}} v_{r}^{2}, \\
\sigma v_{r}\left(X_{1}^{+} X_{1}^{-} \rightarrow \bar{f} f\right)=\frac{e^{4} n_{c}^{f}\left[c_{W}^{2}-2 c_{W} s_{W} q^{f} g_{V}^{f}+s_{W}^{2}\left(g_{A}^{f 2}+g_{V}^{f 2}\right)\right]}{96 \pi c_{W}^{2} m_{X_{1}}^{2}} v_{r}^{2},
\end{gathered}
$$


Here, $q^{f}$ is the electric charge of the SM fermion; $g_{A}^{f}\left(g_{V}^{f}\right)$ is the axi-vector (vector) couplings of the $Z$ to the SM fermion up to the electric coupling $e ; n_{c}^{f}=3$ for quarks and 1 for leptons. To derive the above formulas, we have only included the leading terms in $v_{r}$ for each equation. If the charged particle were stable, its thermal relic abundance should be related to its mass by $\Omega_{X_{1}} h^{2}=0.11 \times m_{X_{1}} /(750 \mathrm{GeV}) .{ }^{5}$ To derive this mass, we have found that the $p$-wave suppressed annihilation cross section or the terms at $\mathcal{O}\left(v_{r}^{2}\right)$ is subdominant compared to the total annihilation cross section.

For a lifetime of $X_{1}^{ \pm}$not too long in the cosmological time scale, we should anticipate that $X_{1}^{+}$has already decayed into its daughter particle and the final dark matter in our current universe is composed of $\chi$. On the other hand, the lifetime of $X_{1}^{ \pm}$can not be too short. Otherwise, the produced $\chi$ particles from $X_{1}^{ \pm}$decays in the early universe can easily annihilate away and do not provide enough dark matter energy density. To calculate the thermal history of the $\chi$ field, one needs to solve for the following coupled Boltzmann equations between $\chi$ and $X_{1}^{ \pm}$

$$
\begin{gathered}
\frac{d n_{X_{1}}}{d t}+3 H n_{X_{1}}=-\langle\sigma v\rangle_{X_{1}}\left(n_{X_{1}}^{2}-n_{X_{1}}^{\mathrm{eq} 2}\right)-n_{X_{1}} \Gamma_{X_{1}}, \\
\frac{d n_{\chi}}{d t}+3 H n_{\chi}=-\langle\sigma v\rangle_{\chi}\left(n_{\chi}^{2}-n_{\chi}^{\mathrm{eq} 2}\right)+n_{X_{1}} \Gamma_{X_{1}} .
\end{gathered}
$$

Here, in the radiation dominated era, $H=\left(8 \pi \rho / 3 M_{\mathrm{Pl}}\right)^{1 / 2}, t=1 /(2 H), \rho(T)=g_{*} \pi^{2} T^{4} / 30$, and $n_{i}^{\mathrm{eq}}(T)=g_{i}\left(m_{i} T / 2 \pi\right)^{3 / 2} e^{-m_{i} / T}$, where $g_{*}=86.25$ is the number of degrees of relativistic freedom and $g_{\chi}=4$ and $g_{X_{1}}=2$. It is convenient to rescale the number density by the entropy and to define the quantity $Y_{i} \equiv n_{i} / s$ with $s=2 \pi^{2} g_{*} T^{3} / 45$. The coupled equations become

$$
\begin{aligned}
\frac{d Y_{X_{1}}}{d x} & =-\frac{s\langle\sigma v\rangle_{X_{1}}}{H x}\left(Y_{X_{1}}^{2}-Y_{X_{1}}^{\mathrm{eq} 2}\right)-\frac{\Gamma_{X_{1}} Y_{X_{1}}}{H x}, \\
\frac{d Y_{\chi}}{d x} & =-\frac{s\langle\sigma v\rangle_{\chi}}{H x}\left(Y_{\chi}^{2}-Y_{\chi}^{\mathrm{eq} 2}\right)+\frac{\Gamma_{X_{1}} Y_{X_{1}}}{H x},
\end{aligned}
$$

where $x \equiv m_{\chi} / T$ and $d x / d t=H x$. The final $\chi$ relic abundance is given by $\Omega_{\chi}=\rho_{\chi} / \rho_{c}$, where $\rho_{c}=3 H_{0}^{2} M_{\mathrm{Pl}}^{2} / 8 \pi=1.0539 \times 10^{-5} h^{2} \mathrm{GeV} \mathrm{cm}^{-3}$ is the critical density corresponding to a flat universe and $\rho_{\chi}=m_{\chi} s_{0} Y_{\chi}(\infty)$ with $s_{0}=2889.2 \mathrm{~cm}^{-3}$ being the entropy today.

At the temperature region with $\mathcal{O}(20)<x<1000$, the decaying terms in eq. (3.21) and (3.22) are not important. The number densities of $X_{1}$ and $\chi$ reach their separate freeze-out values. Since the cross section of $\chi$ is much larger than $X_{1}$, the freeze-out number density for $\chi$ is much below the one of $X_{1}$. At a later time, only the last terms in eq. (3.21) and (3.22) become important. One can easily show that the quantity $Y_{X_{1}}+Y_{\chi}$ is a conserved number. As a result, the final number density of $\chi$ should just match to the number density of $X_{1}$ at $\mathcal{O}(20)$. So, approximately we have the relic abundance of $\chi$ as

$$
\Omega_{\chi} h^{2} \approx 0.11 \times \frac{m_{\chi}}{135 \mathrm{GeV}} \times \frac{m_{X_{1}}}{3.8 \mathrm{TeV}},
$$

\footnotetext{
${ }^{5}$ Here we take $\Omega_{X_{1}} h^{2}=0.11$ as a reference point. In the later calculation, we will choose the right $X_{1}$ mass to provide a correct dark matter thermal relic abundance.
} 


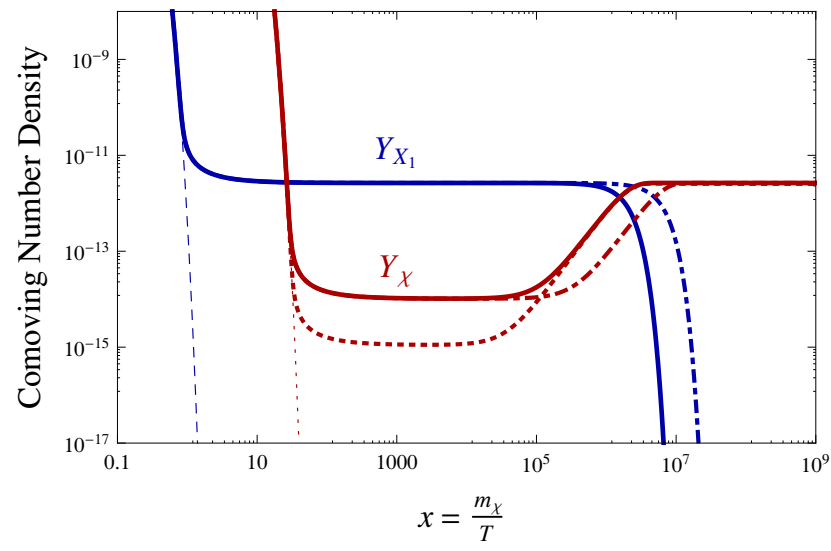

Figure 5. The comoving number density as a function of the temperature. Here, we choose $m_{\chi}=135 \mathrm{GeV}$ and $m_{X_{1}}=3.8 \mathrm{TeV}$. The annihilation cross section of $X_{1}$, approximately $0.03 \mathrm{pb}$, is determined by its interactions with electroweak gauge bosons. The solid lines are for $\left\langle\sigma v_{r}\right\rangle_{\chi}=10 \mathrm{pb}$ and $\tau_{X_{1}}=10 \mathrm{~s}$; the dotdashed lines are for $\left\langle\sigma v_{r}\right\rangle_{\chi}=10 \mathrm{pb}$ and $\tau_{X_{1}}=100 \mathrm{~s}$; the dotted and red line is for $\left\langle\sigma v_{r}\right\rangle_{\chi}=100 \mathrm{pb}$ and $\tau_{X_{1}}=10 \mathrm{~s}$. The relic abundance of $\chi$ satisfies the observed dark matter energy density, $\Omega_{\chi} h^{2}=0.11$.

So, the charged particle $X_{1}$ is predicted to be $3.8 \mathrm{TeV}$ and the other charged particle $X_{2}$ should be below around $1 \mathrm{TeV}$ to explain the ratio of INTEGRAL and Fermi-LAT cross sections in eq. (3.12).

We solve the coupled equations in eq. (3.22) numerically and show both comoving number densities of $\chi$ and $X_{1}$ in figure 5. We find that if the mass of $X_{1}$ is $3.8 \mathrm{TeV}$, it generates the relic abundance for $\chi$ which satisfies the total dark matter energy density, $\Omega_{\chi} h^{2}=0.11$. In the blue solid and the red solid lines, for $\left\langle\sigma v_{r}\right\rangle_{\chi}=10 \mathrm{pb}^{6}$ and $\tau_{X_{1}}=$ $10 \mathrm{~s}$ we show the evolutions of the $X_{1}$ and $\chi$ comoving number densities as a function of temperature. As can be seen from figure 5 and at $x \approx 20$, both $X_{1}$ and $\chi$ have reached ordinary relic abundances according to their respective annihilation cross sections. At $x \approx 10^{5}-10^{6}, X_{1}$ starts to decay and its number density drops rapidly. Meanwhile, the stable $\chi$ particle number density increases and reaches a plateau at around $10^{7}$. The final number density of the $\chi$ field is found to be independent on the lifetime $\tau_{X_{1}}$, as long as the decay happens late enough so that the annihilation of $\chi$ is not important any more. The actual time for $\chi$ to reach its eventual number density is proportional to $\sqrt{\tau_{X_{1}}}$.

To satisfy the dark matter relic abundance, the lifetime of the charged particle $X_{1}$ can be $\leq 100 \mathrm{~s}$. For such a late decayed particle, we need to worry about its modification on the Big Bang nucleosynthesis (BBN) history. Since the main decaying product of $X_{1}$ is into leptons plus the stable $\chi$, the BBN constrains are fairly weak. From ref. [58], the ${ }^{6} \mathrm{Li} /{ }^{7} \mathrm{Li}$ ratio constrains the lifetime of $X_{1}$ to be $\tau_{X_{1}}<10^{5} s$ for $\Omega_{X_{1}} h^{2} \approx 0.5$ if it would have

\footnotetext{
${ }^{6}$ The annihilation cross section of $\chi$ is not necessarily related to its annihilation cross section at the current time. This is because its main production here is from the heavy particle $X_{1}$ decay, and it has a relativistic velocity and hence a smaller cross section.
} 
not decayed. As pointed in ref. [59, 60], the long-lived charged particle, with a lifetime $\tau_{X_{1}}>10^{3} \mathrm{~s}$, can form a bound state with nuclei and enhance the ${ }^{6} \mathrm{Li}$ production. The parameter space in our model can indeed satisfy the BBN constraints.

\section{Discussion and conclusions}

The charged particle $X_{1}$ in our model behaves as a heavy stable charged particle (HSCP) at colliders. The current searches from CMS at $\sqrt{s}=7 \mathrm{TeV}$ and $5.0 \mathrm{fb}^{-1}$ have set a lower limit on the mass of $X_{1}$ to be $223 \mathrm{GeV}$ at $95 \%$ C.L. [61]. For the predicted mass of $X_{1}$ around $3.8 \mathrm{TeV}$, the existing studies have shown that the $14 \mathrm{TeV}$ LHC with $100 \mathrm{fb}^{-1}$ can reach the HSCP up to a mass around $1 \mathrm{TeV}$. So, unlikely the stable charged particle can be discovered at the $14 \mathrm{TeV}$ LHC. However, for the other charged particle $X_{2}$ its mass should be below $1 \mathrm{TeV}$ and could be a long-lived particle or decay into SM particles, for instance $X_{2}^{+} \rightarrow e^{+} \nu_{e}$. The parameter space of the $X_{2}$ particle will be well explored at the LHC $14 \mathrm{TeV}$ running.

One feature of our model is directly using photon as a mediator to link the dark matter sector to positron/electron. Unfortunately, other than searching for the charged particles responsible for the charge radius operator, in the near future there is no additional observable dark matter direct or indirect signatures for the $\chi$ field, which has interactions with SM particles suppressed by the $\mathrm{TeV}$ scale cutoff of the effective operators. The minor component of dark matter, $\phi_{1}$, may have detectable effects. However, that highly relies on the parameters one chooses, thus we do not pursue that in detail here. Another ingredient that we utilize is the $s$-channel resonance particle to increase the annihilation cross section. We want to stress that this option is not a unique one and is introduced just for convenience. One can also introduce a light mediator in the $t$-channel plus the Sommerfeld enhancement to achieve the same goal [54].

In summary, we have constructed a realistic model to have the same dark matter particle responsible for both the INTEGRAL $511 \mathrm{keV}$ and Fermi-LAT $135 \mathrm{GeV}$ lines. Through an $s$-channel resonance, the dark matter particles annihilate into a complex scalar, which couples to photon via a charge-radius operator. For a few $\mathrm{MeV}$ mass splitting between the real and imaginary parts of the complex scalar, two pairs of electron and positron are the main visible particles from dark matter annihilation. We have worked out the parameter space and have found that both the large cross section required for INTEGRAL and the small cross section for Fermi-LAT can be simultaneously accommodated in our model. The thermal relic abundance of dark matter is achieved by the late decay of a charged particle, which also generates the charge-radius operator. The other charged particle responsible for the charge-radius operator is predicted to have a mass below $1 \mathrm{TeV}$. The $14 \mathrm{TeV}$ LHC will concretely test the scenario presented in this paper.

\section{Acknowledgments}

We would like to thank James Cline, Tim Cohen, Douglas Finkbeiner, JoAnne Hewett, Dan Hooper, Jessie Shelton, Tracy Slatyer, Aaron Vincent and Jay Wacker for useful discussions 
and comments. YB is supported by start-up funds from the University of Wisconsin, Madison. YB thanks SLAC for their warm hospitality. SLAC is operated by Stanford University for the US Department of Energy under contract DE-AC02-76SF00515. We also thank the Aspen Center for Physics, under NSF Grant No. 1066293, where part of this work was completed. Support for the work of M.S. was provided by NASA through Einstein Postdoctoral Fellowship grant number PF2-130102 awarded by the Chandra X-ray Center, which is operated by the Smithsonian Astrophysical Observatory for NASA under contract NAS8-03060.

Open Access. This article is distributed under the terms of the Creative Commons Attribution License which permits any use, distribution and reproduction in any medium, provided the original author(s) and source are credited.

\section{References}

[1] L. Bergstrom and P. Ullio, Full one loop calculation of neutralino annihilation into two photons, Nucl. Phys. B 504 (1997) 27 [hep-ph/9706232] [INSPIRE].

[2] Z. Bern, P. Gondolo and M. Perelstein, Neutralino annihilation into two photons, Phys. Lett. B 411 (1997) 86 [hep-ph/9706538] [INSPIRE].

[3] L. Bergstrom, P. Ullio and J.H. Buckley, Observability of gamma-rays from dark matter neutralino annihilations in the Milky Way halo, Astropart. Phys. 9 (1998) 137 [astro-ph/9712318] [INSPIRE].

[4] P. Ullio and L. Bergstrom, Neutralino annihilation into a photon and a $Z$ boson, Phys. Rev. D 57 (1998) 1962 [hep-ph/9707333] [INSPIRE].

[5] M. Perelstein and A. Spray, Indirect detection of little Higgs dark matter, Phys. Rev. D 75 (2007) 083519 [hep-ph/0610357] [INSPIRE].

[6] G. Bertone, C. Jackson, G. Shaughnessy, T.M. Tait and A. Vallinotto, Gamma ray lines from a universal extra dimension, JCAP 03 (2012) 020 [arXiv: 1009.5107] [INSPIRE].

[7] W.N. Johnson III, F.R. Harnden Jr. and R.C. Haymes, The spectrum of low-energy gamma radiation from the galactic-center region, Astrophys. J. 172 (1972) L1.

[8] W.N. Johnson III and R.C. Haymes, Detection of a gamma-ray spectral line from the galactic-center region, Astrophys. J. 184 (1973) 103.

[9] R.C. Haymes, G.D. Walraven, C.A. Meegan, R.D. Hall, F.T. Djuth and D.H. Shelton, Detection of nuclear gamma rays from the galactic center region, Astrophys. J. 201 (1975) 593.

[10] M. Leventhal, C.J. MacCallum and P.D. Stang, Detection of $511 \mathrm{keV}$ positron annihilation radiation from the galactic center direction, Astrophys. J. 225 (1978) L11.

[11] R.W. Bussard, R. Ramaty and R.J. Drachman, The annihilation of galactic positrons, Astrophys. J. 228 (1979) 928.

[12] Y. Ascasibar, P. Jean, C. Boehm and J. Knoedlseder, Constraints on dark matter and the shape of the milky way dark halo from the $511 \mathrm{keV}$ line,

Mon. Not. Roy. Astron. Soc. 368 (2006) 1695 [astro-ph/0507142] [INSPIRE]. 
[13] E. Churazov, R. Sunyaev, S. Sazonov, M. Revnivtsev and D. Varshalovich, Positron annihilation spectrum from the galactic center region observed by SPI/INTEGRAL, Mon. Not. Roy. Astron. Soc. 357 (2005) 1377 [astro-ph/0411351] [INSPIRE].

[14] G. Weidenspointner et al., The sky distribution of positronium annihilation continuum emission measured with spi/integral, Astron. Astrophys. 450 (2006) 1013 [astro-ph/0601673] [INSPIRE].

[15] J.F. Beacom and H. Yuksel, Stringent constraint on galactic positron production, Phys. Rev. Lett. 97 (2006) 071102 [astro-ph/0512411] [INSPIRE].

[16] N. Prantzos et al., The $511 \mathrm{keV}$ emission from positron annihilation in the galaxy, arXiv: 1009.4620 [INSPIRE].

[17] C. Picciotto and M. Pospelov, Unstable relics as a source of galactic positrons, Phys. Lett. B 605 (2005) 15 [hep-ph/0402178] [INSPIRE].

[18] D. Hooper and L.-T. Wang, Possible evidence for axino dark matter in the galactic bulge, Phys. Rev. D 70 (2004) 063506 [hep-ph/0402220] [INSPIRE].

[19] C. Boehm, D. Hooper, J. Silk, M. Casse and J. Paul, MeV dark matter: has it been detected?, Phys. Rev. Lett. 92 (2004) 101301 [astro-ph/0309686] [INSPIRE].

[20] M. Pospelov, A. Ritz and M.B. Voloshin, Secluded WIMP dark matter, Phys. Lett. B 662 (2008) 53 [arXiv:0711.4866] [inSPIRE].

[21] D. Hooper and K.M. Zurek, A natural supersymmetric model with MeV dark matter, Phys. Rev. D 77 (2008) 087302 [arXiv:0801.3686] [INSPIRE].

[22] J.-H. Huh, J.E. Kim, J.-C. Park and S.C. Park, Galactic 511 keV line from MeV milli-charged dark matter, Phys. Rev. D 77 (2008) 123503 [arXiv: 0711.3528] [INSPIRE].

[23] M. Pospelov and A. Ritz, The galactic 511 keV line from electroweak scale WIMPs, Phys. Lett. B 651 (2007) 208 [hep-ph/0703128] [INSPIRE].

[24] D.P. Finkbeiner and N. Weiner, Exciting dark matter and the INTEGRAL/SPI $511 \mathrm{keV}$ signal, Phys. Rev. D 76 (2007) 083519 [astro-ph/0702587] [INSPIRE].

[25] N. Arkani-Hamed, D.P. Finkbeiner, T.R. Slatyer and N. Weiner, A theory of dark matter, Phys. Rev. D 79 (2009) 015014 [arXiv:0810.0713] [InSPIRE].

[26] F. Chen, J.M. Cline and A.R. Frey, A new twist on excited dark matter: implications for INTEGRAL, PAMELA/ATIC/PPB-BETS, DAMA, Phys. Rev. D 79 (2009) 063530 [arXiv: 0901.4327] [INSPIRE].

[27] D.P. Finkbeiner, T.R. Slatyer, N. Weiner and I. Yavin, PAMELA, DAMA, INTEGRAL and signatures of metastable excited WIMPs, JCAP 09 (2009) 037 [arXiv:0903.1037] [INSPIRE].

[28] F. Chen, J.M. Cline and A.R. Frey, Nonabelian dark matter: models and constraints, Phys. Rev. D 80 (2009) 083516 [arXiv:0907.4746] [INSPIRE].

[29] J.M. Cline, A.R. Frey and F. Chen, Metastable dark matter mechanisms for INTEGRAL $511 \mathrm{keV} \gamma$ rays and DAMA/CoGeNT events, Phys. Rev. D 83 (2011) 083511 [arXiv: 1008.1784] [INSPIRE].

[30] J. Cline and A. Frey, Abelian dark matter models for $511 \mathrm{keV}$ gamma rays and direct detection, Annalen Phys. 524 (2012) 579 [arXiv:1204.1965] [InSPIRE].

[31] D. Feldman, Z. Liu and P. Nath, PAMELA positron excess as a signal from the hidden sector, Phys. Rev. D 79 (2009) 063509 [arXiv:0810.5762] [INSPIRE]. 
[32] F. Chen, J.M. Cline, A. Fradette, A.R. Frey and C. Rabideau, Exciting dark matter in the galactic center, Phys. Rev. D 81 (2010) 043523 [arXiv:0911.2222] [INSPIRE].

[33] R. Morris and N. Weiner, Low energy INTEGRAL positrons from exciting dark matter, arXiv:1109.3747 [INSPIRE].

[34] C. Weniger, A tentative gamma-ray line from dark matter annihilation at the Fermi Large Area Telescope, JCAP 08 (2012) 007 [arXiv: 1204.2797] [INSPIRE].

[35] T. Bringmann, X. Huang, A. Ibarra, S. Vogl and C. Weniger, Fermi LAT search for internal bremsstrahlung signatures from dark matter annihilation, JCAP 07 (2012) 054 [arXiv: 1203.1312] [INSPIRE].

[36] M. Su and D.P. Finkbeiner, Strong evidence for gamma-ray line emission from the inner galaxy, arXiv:1206.1616 [INSPIRE].

[37] Fermi-LAT collaboration, Search gamma-ray spectral lines in the milky way diffuse with the Fermi Large Area Telescope, in The Fermi symposium, Monterey U.S.A. October 28November 22012.

[38] T. Bringmann and C. Weniger, Gamma ray signals from dark matter: concepts, status and prospects, Phys. Dark Univ. 1 (2012) 194 [arXiv: 1208.5481] [INSPIRE].

[39] W. Buchmüller and M. Garny, Decaying vs annihilating dark matter in light of a tentative gamma-ray line, JCAP 08 (2012) 035 [arXiv:1206.7056] [INSPIRE].

[40] L. Bouchet, J.-P. Roques and E. Jourdain, On the morphology of the electron-positron annihilation emission as seen by SPI/INTEGRAL, Astrophys. J. 720 (2010) 1772 [arXiv:1007.4753] [INSPIRE].

[41] M. Su and D.P. Finkbeiner, Double gamma-ray lines from unassociated Fermi-LAT sources, arXiv: 1207.7060 [INSPIRE].

[42] G. Jungman, M. Kamionkowski and K. Griest, Supersymmetric dark matter, Phys. Rept. 267 (1996) 195 [hep-ph/9506380] [INSPIRE].

[43] P. Jean et al., Spectral analysis of the galactic $e^{+} e^{-}$annihilation emission, Astron. Astrophys. 445 (2006) 579 [astro-ph/0509298] [INSPIRE].

[44] J.F. Navarro et al., The inner structure of $\Lambda C D M$ halos 3: universality and asymptotic slopes, Mon. Not. Roy. Astron. Soc. 349 (2004) 1039 [astro-ph/0311231] [INSPIRE].

[45] J.F. Navarro, C.S. Frenk and S.D. White, A universal density profile from hierarchical clustering, Astrophys. J. 490 (1997) 493 [astro-ph/9611107] [INSPIRE].

[46] A.C. Vincent, P. Martin and J.M. Cline, Interacting dark matter contribution to the galactic $511 \mathrm{keV}$ gamma ray emission: constraining the morphology with INTEGRAL/SPI observations, JCAP 04 (2012) 022 [arXiv:1201.0997] [INSPIRE].

[47] M. Ibe, H. Murayama and T. Yanagida, Breit-Wigner enhancement of dark matter annihilation, Phys. Rev. D 79 (2009) 095009 [arXiv:0812.0072] [InSPIRE].

[48] H.M. Lee, M. Park and W.-I. Park, Fermi gamma ray line at $130 \mathrm{GeV}$ from axion-mediated dark matter, Phys. Rev. D 86 (2012) 103502 [arXiv:1205.4675] [INSPIRE].

[49] M.R. Buckley and D. Hooper, Implications of a $130 \mathrm{GeV}$ gamma-ray line for dark matter, Phys. Rev. D 86 (2012) 043524 [arXiv: 1205.6811] [INSPIRE].

[50] H.M. Lee, M. Park and W.-I. Park, Axion-mediated dark matter and Higgs diphoton signal, JHEP 12 (2012) 037 [arXiv:1209.1955] [INSPIRE]. 
[51] Y. Bai and J. Shelton, Gamma lines without a continuum: thermal models for the Fermi-LAT 130 GeV gamma line, JHEP 12 (2012) 056 [arXiv: 1208.4100] [INSPIRE].

[52] G. Chalons, M.J. Dolan and C. McCabe, Neutralino dark matter and the Fermi gamma-ray lines, arXiv:1211.5154 [INSPIRE].

[53] K. Schmidt-Hoberg, F. Staub and M.W. Winkler, Enhanced diphoton rates at Fermi and the LHC, JHEP 01 (2013) 124 [arXiv:1211.2835] [INSPIRE].

[54] N. Arkani-Hamed, D.P. Finkbeiner, T.R. Slatyer and N. Weiner, A theory of dark matter, Phys. Rev. D 79 (2009) 015014 [arXiv:0810.0713] [INSPIRE].

[55] R.E. Shrock and M. Suzuki, Invisible decays of Higgs bosons, Phys. Lett. B 110 (1982) 250 [INSPIRE].

[56] V. Barger, P. Langacker, M. McCaskey, M.J. Ramsey-Musolf and G. Shaughnessy, LHC phenomenology of an extended standard model with a real scalar singlet, Phys. Rev. D 77 (2008) 035005 [arXiv:0706.4311] [InSPIRE].

[57] C. Burgess, M. Pospelov and T. ter Veldhuis, The Minimal model of nonbaryonic dark matter: a singlet scalar, Nucl. Phys. B 619 (2001) 709 [hep-ph/0011335] [INSPIRE].

[58] K. Jedamzik, Big bang nucleosynthesis constraints on hadronically and electromagnetically decaying relic neutral particles, Phys. Rev. D 74 (2006) 103509 [hep-ph/0604251] [INSPIRE].

[59] M. Pospelov, Particle physics catalysis of thermal big bang nucleosynthesis, Phys. Rev. Lett. 98 (2007) 231301 [hep-ph/0605215] [INSPIRE].

[60] K. Kohri and F. Takayama, Big bang nucleosynthesis with long lived charged massive particles, Phys. Rev. D 76 (2007) 063507 [hep-ph/0605243] [INSPIRE].

[61] CMS collaboration, Search for heavy long-lived charged particles in pp collisions at $\sqrt{s}=7 \mathrm{TeV}$, Phys. Lett. B $\mathbf{7 1 3}$ (2012) 408 [arXiv: 1205.0272] [INSPIRE]. 